\title{
El puño invisible. Arte, revolución y un siglo de cambios culturales
}

\section{Carlos Arturo Robledo-Marín}

Doctor en Humanidades. Licenciado en Educación

Fundación Opción Colombia - FUNDACOL. Medellín, Colombia https:/ / orcid.org/0000-

0002-6944-561X・direccionejecutiva@fundacol.com

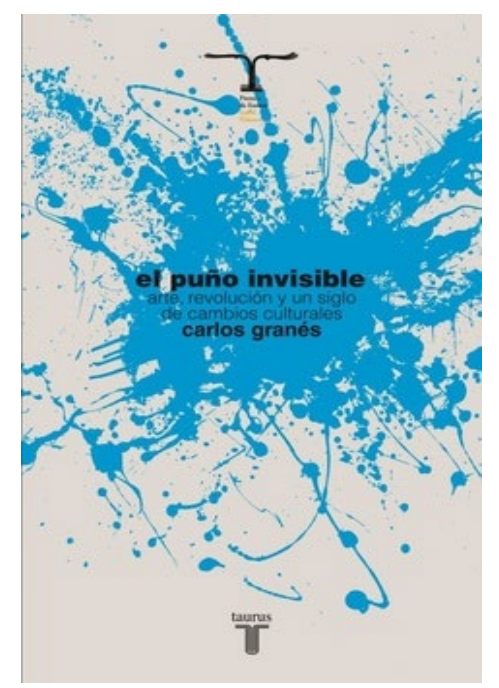

Granés, Carlos. 2012.

El puño invisible. Arte, revolución y un siglo de cambios culturales.

Bogotá, Colombia. Editorial Taurus.

$492 \mathrm{p}$.

ISBN: 978-958-758-394-6

La metáfora del puño invisible es utilizada por el antropólogo colombiano Carlos Granés para trazar el hilo conductor de su narración, en la que argumenta cómo el germen vanguardista sobrevive al horror del nazismo, transformándose, de generación en generación, hasta lograr de forma silenciosa, invisible, una trasmutación de los valores. El libro El Puño invisible responde algunos interrogantes con relación a las transformaciones de la revolución cultural en el último siglo “¿Cómo se gestó la revolución cultural que ha moldeado las sociedades contemporáneas? ¿Cómo se transmitió de generación en generación? ¿Cuáles han sido las consecuencias de su triunfo?” (Granés, 2012, p. 14).

Recibido: 04/04/2020 | Aprobado: 07/09/2020 |Publicado: 01/01/2021

\section{¿Cómo citar este artículo? / How to quote this article?}

Robledo-Marín, C. A. (2021). El puño invisible. Arte, revolución y un siglo de cambios culturales. Prospectiva. Revista de Trabajo Social e intervención social, (31), 475-478. doi: https:// doi.org/10.25100/ prts.v0i31.9392. 
El propósito central del autor es evidenciar el triunfo silencioso de la revolución cultural de vanguardia sobre la revolución social del comunismo que pierde su fuerza a mediados de los ochenta. La primera con sus ideas libertarias y vanguardistas, que promulgaban el deseo individual y la transformación de la vida en arte, se antepone ante la segunda con su ideología marxista de desmantelar las estructuras de los Estados, alterar su economía y administración del poder. Para el autor, el triunfo de la revolución cultural, se convierte a su vez en su derrota, pues el discurso anárquico de la vanguardia es asimilado por la sociedad, enaltecido por el mercado y los medios de comunicación, dejando a un lado la primigenia actitud de rebeldía y virulencia, características de la vanguardia, para dar paso a una actitud generalizada e inofensiva.

Para dar desarrollo a los argumentos, la obra se sitúa en el contexto histórico, la cual tiene como eje central la gestación y transformación de la revolución cultural de Europa y Estados Unidos. Además se divide en dos partes: un nutrido primer tiempo donde hace un recorrido a través de la influencia de diversos personajes, movimientos de vanguardia, corrientes culturales, movimientos artísticos y literarios, tales como: futuristas, dadaístas, surrealistas, letristas, músicos experimentales, poetas beat, situacionistas, yippies y demás revolucionarios culturales y un segundo tiempo, más corto, donde relacionan estos hechos con sus respectivas consecuencias.

El primer tiempo describe de manera detallada cómo se da el logro de la revolución cultural sobre la revolución política, gracias al aporte de diversas vanguardias, siendo Marinetti un precursor visionario, al instar la eliminación del pasado y todas las estructuras que lo representaran, invocando así la destrucción, el cambio, la descomposición del lenguaje y la música en ruidos para dar paso a lo novedoso, al futuro, a la creación de un hombre nuevo. Estas premisas son retomadas por el dadaísmo, para enriquecerlo con el absurdo y el nihilismo, siendo Tzara y sus colegas quienes logran ganar adeptos con sus propuestas de crear estilos de vida que fueran una obra de arte en si mismo. Breton por su parte, retoma elementos de estas dos vanguardias y le insufla la irracionalidad para la alteración de la conciencia y dar paso al "hedonismo, el erotismo y la existencia como una empresa creadora" (p. 461). Posteriormente, los jóvenes, la transformación de la ciudad y la superación del arte retoman importancia con el letrismo de Isou y los pensamientos de Debord donde tanto en Europa como en los Estados Unidos, se fortalecen las ideas de la necesidad de una revolución en la vida cotidiana, despertar del mutismo y anquilosamiento del trabajo para dar paso a la aventura, a la toma de las calles, donde lo personal se vuelve político: Fornicar, drogarse y divertirse, conjugado con la alucinación, la diversión, la ocurrencia y el ocio se convierten en las mejores armas para atacar frontalmente el poder del capitalismo y a la sociedad alienante. 
Es así, como en el primer tiempo se logra una revolución cultural del arte, las ideas y la política. Por un lado, la fuerza revolucionaria de la juventud desmonta las pacatas formas de la sociedad del momento y se revindica como un colectivo visible con sus deseos de libertad y por otro lado, se desacralizan las grandes obras del pasado, se destruye la alta cultura y los valores que la sustentan, se resignifica la vida y los objetos cotidianos como obra de arte, siendo la experimentación y la imaginación la plataforma de la transformación del hombre y la sociedad. Por tanto, lo museos, las galerías y teatros pierden su valor, pues lo realmente importante para contemplar estaba en las calles, en la vida misma, creándose así una nueva escala de valores, una manera de vivir revolucionaria donde la generación de espacios para la expresión del yo atacaba frontalmente los márgenes de la libertad individual, derrocando tabúes, jerarquías, dando paso a su vez al hedonismo, la irreverencia y la frivolidad. En suma, el primer tiempo trastocaría el sistema completamente.

En el segundo tiempo, el autor argumenta cómo lo descrito líneas arriba propicia una revolución cultural, mas no política, dado que esta no logra el derrocamiento del sistema, ni la muerte del arte, ni del museo, ni forja una nueva era sin patronos ni burgueses. Asimismo, en el segundo tiempo, las nociones de revolución y rebeldía pierden su esencia virulenta, al dejar de ser prácticas marginales para convertirse en el nuevo paradigma social, caracterizado por el individualismo y el culto al yo. Ya no hay nada que destruir, pues la sociedad de finales de los sesenta y principios de los setenta cambia dramáticamente, asumiendo los valores de la contracultura, así las cosas la revolución pierde su poder de transformación social.

Esta revolución descafeinada, como la nombra el autor, abre paso a una revolución mediática, puesto que la revolución cultural de otrora pierde su envoltorio transgresor para revelar su trasfondo banal: responder a las nuevas demandas de la sociedad, a través de la rebeldía, la diversión y la vida sin responsabilidades. El capitalismo ve en la revolución mediática una gran oportunidad para convertir al ser humano en un insaciable consumidor de rebeldía, y no la desperdicia, por el contrario, se reinventa a sí mismo para no ser inferior a la demanda; en este orden de ideas la moda también se adapta a los cambios, asume el lenguaje de vanguardia, transforma los productos burgueses y anticuados en una nueva tendencia que será el eje de la moda hasta el momento: la rebeldía; por su lado, los medios de comunicación se fortalecen como una industria de entretenimiento y ocio, el formato televisivo encarna los elementos cínicos y escépticos de la vanguardia sin dificultad; la política se apropia del discurso de la contracultura para ganar adeptos; el arte en lugar de oponerse a la sociedad en la que vive, manteniendo su carácter revolucionario y anti institucional, se mercantiliza, el artista se focaliza en la disputa por el poder, el reconocimiento institucional y la aprobación de los curadores. Cualquier cosa se convierte en arte, siempre y cuando genere suficiente expectativa y escándalo como para llamar la atención del museo, y aunque muchas obras se muestren transgresoras y rebeldes, Granés sostiene que, hoy por hoy, se vive en un periodo de calma cultural, donde prevalecen la 
Robledo-Marín

frivolidad y la inocuidad de las obras. Es así, como en este segundo tiempo la rebelión se convierte en algo popular e inocuo, pues la rebeldía es el lenguaje del mercado y de los medios de comunicación, siempre a la búsqueda de lo nuevo, lo último, lo trasgresor, lo sensacional.

El libro, El puño invisible, podría motivar en la comunidad académica proyectos de investigación entre los cuales conviene destacar: las relaciones entre Arte, Ciencias Sociales y Humanidades; los aportes que el arte puede realizar a la política y la cultura, dado su pluralismo y tolerancia donde todos pese a su diferencia tienen cabida en él; el análisis del arribo de estudios culturales en Latinoamérica; el espacio del arte como asunto policéntrico y no eurocéntrico; el papel del arte contemporáneo en la sociedad actual y su futuro en la sociedad; además del rol de los artistas y escritores para la creación de nuevas corrientes de pensamiento.

\section{Referencias bibliográficas}

Granés, C. (2012). El puño invisible. Arte, revolución y un siglo de cambios culturales. Bogotá, Colombia. Editorial Taurus. 


\section{OTROS ARTÍCULOS DE PROSPECTIVA No. 31 DE 2021}

\section{PRESENTACIÓN}

Presentación. Reflexiones sobre desafios al publicar sistematizaciones

Rosa María Cifuentes-Gil

\section{EDITORIAL}

Reflexiones sobre Trabajo Social: aportes de la Sistematización

María Rocío Cifuentes-Patiño

\section{ARTÍCULOS}

Hacer lo que se sabe, pensar lo que se hace. La sistematización como modalidad investigativa Alfonso Torres-Carrillo

Aportes y desafios de la Sistematización de experiencias en el Trabajo Social y la extensión crítica. Apuntes y reflexiones desde la perspectiva de la Educación Popular

María Rosa Goldar

Valeria Chiavetta

La sistematización en Trabajo Social y la epistemología feminista del punto de vista. Diálogos sobre la producción de conocimiento sustentada en experiencias

Ruth Noemí Parola

María Florencia Linardelli

La Sistematización investigativa de las experiencias: del baile de los que sobran a la fiesta de los que faltan

María Belén Ortega-Senet

Sistematización y Trabajo Social en Chile. El largo y sinuoso camino

Patricia Lorena Castañeda-Meneses

Ana María Salamé-Coulon
Sentipensar la pandemia COVID-19 desde la sistematización de la experiencia en Trabajo Social: reflexiones del profesor Oscar Jara Holliday

Elia Sepúlveda-Hernández

La sistematización de experiencias, una investigación social cualitativa que potencia buenas prácticas de convivencia y gobierno. La experiencia de un conjunto residencial multifamiliar en Cali, Colombia Martha Lucia Echeverry-Velásquez Manuela Prada-Dávila

Construcción de subjetividades epistemológicaspolíticas de profesoras y profesores de Investigación social en una universidad privada y confesional en Bogotá

Giovanni Mora-Lemus

Sistematización de la experiencia Reconocimiento de los derechos humanos del adulto mayor en dos familias residentes en Cali y Valledupar (Colombia)

Lina María Cuello-Lacouture

Jimena del Pilar Jaramillo-Jaramillo

La memoria transformadora como estrategia de intervención profesional en los procesos de reconciliación social: comprensión a partir de mujeres campesinas, excombatientes y jóvenes en Manizales, Colombia

Yeimmy Stephania Corredor-Sotelo

Juliana Fuertes-Fuertes

Sistematización de una estrategia de educación informal implementada en personas privadas de la libertad en el establecimiento penitenciario de mediana seguridad y carcelario de Barranquilla, Colombia

Rafael Humberto Herrera-Mercado Rafael Alberto Zambrano-Vanegas 
Aportes significativos del proceso de intervención comunitaria con la Escuela Popular de Comunicación Alternativa Jaime Garzón de la ciudad de Cúcuta, Colombia

Carlos Lasso-Urbano

La sistematización de la intervención como metodología de investigación en Trabajo Social. Importancia práctica y teórica de la fase de recogida de datos en la intervención social según experiencia del Programa de Apoyo a las Familias en Zaragoza, España

Elisa Esteban-Carbonell

Nuria Del Olmo-Vicén

Papel de la sistematización de experiencias en los procesos de evaluación de intervenciones de salud pública en la Comuna Saludable por la Paz, Cali - Colombia

Jenny Faisury Peña-Varón

Paola Andrea Marín-Velásquez

Janeth Mosquera-Becerra

Experiencia de intervención social en hogares comunitarios integrales del barrio Alfonso Bonilla Aragón, Cali - Colombia

Julián Alexander Montaño-Cárdenas

Las políticas sociales y el gobierno de la "población indígena". Estrategias y regulaciones en el multiculturalismo chileno

Rodrigo Agustín Navarrete-Saavedra

Representaciones sociales sobre estilo de autoridad y tipos de interacción en cuidadores de residencias de protección infantil en Chile

Marcelo Gallegos-Fuentes
Carmen Gloria Jarpa-Arriagada

Reflexiones sobre inseguridad social y cuestiones penales. Una respuesta estratégica a partir de experiencias de cooperativismo con ex detenidos en Argentina

Analia Elizabeth Otero

Yael Yanina Barrera

Desarrollo y salud: la emergencia de un nuevo paradigma

Jesús María Sánchez-Ordóñez

Trabajo Social en ejercicio libre: la perspectiva profesional en España

Paula Frieiro-Padín

Tamara Fernández-Arias

Rubén González-Rodríguez

\section{RESEÑAS}

Social Work and the City: Urban Themes in 21stCentury Social Work

Felipe Saravia-Cortés

Respuestas del Trabajo Social ante emergencias sociales y problemáticas sociales complejas de México y España

Felipe Saravia-Cortés

El feminismo, el género y la profesionalización del trabajo social en Colombia (1936-2004)

Ambar Oriana Serna-Lombo

El puño invisible. Arte, revolución y un siglo de cambios culturales

Carlos Arturo Robledo-Marín

\section{PROSPECTIVA}

No. $31 \bullet$ ene.-jun. 2021

e-ISSN: 2389-993X • Universidad del Valle 